\title{
The immunogenicity and safety of a reduced PRP- content DTPw-HBV/Hib vaccine when administered according to the accelerated EPI schedule
}

\author{
Sukanta Chatterjee ${ }^{1}$, Sylvan J Rego ${ }^{2}$, Fulton D'Souza ${ }^{2}$, BD Bhatia ${ }^{3}$, Alix Collard ${ }^{4}$, Sanjoy K Datta ${ }^{4}$, \\ Jeanne-Marie Jacquet ${ }^{4}$
}

\begin{abstract}
Background: Combination vaccines improve coverage, compliance and effectively introduce new antigens to mass vaccination programmes. This was a phase III, observer-blind, randomized study of GSK Biologicals diphtheriatetanus-whole cell pertussis vaccine combined with hepatitis B and Haemophilus influenzae type $b$ vaccines, containing a reduced amount of polyribosyl-ribitol-phosphate (PRP) and a DTPw component manufactured at a different site (DTPw-HBV/Hib $2.5[\mathrm{Kft}]$ ). The primary aim of this study was to demonstrate that DTPw-HBV/Hib $2.5[\mathrm{Kft}]$ was not inferior to the licensed DTPw-HBV/Hib (Tritanrix(tm)-HepB/Hiberix(tm)) vaccine or the DTPw-HBV/Hib 2.5 vaccine, also containing a reduced amount of PRP, with respect to the immune response to the PRP antigen, when administered to healthy infants, according to the Expanded Programme for Immunization (EPI) schedule at 6, 10 and 14 weeks of age.

Methods: 299 healthy infants were randomised to receive either DTPw-HBV/Hib $2.5[\mathrm{Kft}] \mathrm{DTPw}-\mathrm{HBV} / \mathrm{Hib} \mathrm{b}_{2.5}$ or DTPwHBV/Hib according to the 6-10-14 week EPI schedule. Blood samples were analysed prior to the first dose of study vaccine and one month after the third vaccine dose for the analysis of immune responses. Solicited local and general symptoms such as pain, redness and swelling at the injection site and drowsiness and fever, unsolicited symptoms (defined as any additional adverse event) and serious adverse events (SAEs) were recorded up to 20 weeks of age.

Results: One month after the third vaccine dose, $100 \%$ of subjects receiving DTPw-HBV/Hib 2.5 [Kft] or DTPw-HBV/ Hib and $98.8 \%$ of subjects receiving DTPw-HBV/Hib 2.5 vaccine had seroprotective levels of anti-PRP antibodies (defined as anti-PRP antibody concentration $\geq 0.15 \mu \mathrm{g} / \mathrm{ml}$ ). Seroprotective antibody concentrations were attained in over $98.9 \%$ of subjects for diphtheria, tetanus and hepatitis B. The vaccine response rate to pertussis antigen was at least $97.8 \%$ in each group. Overall, the DTPw-HBV/Hib 2.5 [Kft] vaccine was well tolerated in healthy infants; no SAEs were reported in any group.
\end{abstract}

Conclusions: The DTPW-HBV/Hib 2.5 [Kft] vaccine was immunogenic and well-tolerated when administered according to the EPI schedule to Indian infants.

Trial registration: http://www.clinicaltrials.gov NCT00473668.

\section{Background}

Combination vaccines improve individual compliance and vaccination coverage and offer a convenient vehicle for introducing community protection against new diseases by adding antigens to an existing vaccine with high coverage $[1,2]$. The diphtheria-tetanus-whole cell

\footnotetext{
* Correspondence: sanjoy.k.datta@gskbio.com

${ }^{4}$ GlaxoSmithKline Biologicals, Bangalore, India and Wavre, Belgium

Full list of author information is available at the end of the article
}

pertussis (DTPw) is one such vaccine, with a global coverage of $81 \%$ in 2007 [3].

In 1996, GlaxoSmithKline (GSK) Biologicals licensed the first combined DTPw and hepatitis B vaccine (DTPw-HBV, Tritanrix (tm)Hep B), which was shown to improve the uptake of hepatitis B vaccine in Thailand [4]. Haemophilus influenzae type $\mathrm{b}$ (Hib) protection was added via the monovalent vaccine, Hiberix (tm) to form DTPw-HBV/Hib. This combination vaccine facilitated 
the introduction of hepatitis $B$ and Hib vaccinations to large parts of the developing world [5].

Increasing global demands for DTPw-based combination vaccines has necessitated creative strategies to ensure the adequate supply of the vaccine antigens, through reducing Hib antigen content and expanding antigen production at new manufacturing sites. Primary vaccination with reduced Hib content vaccines has been shown to confer vaccine response rates, after primary vaccination, at least as high as those observed with commercially available DTPw-HBV/Hib containing $10 \mu \mathrm{g}$ PRP [6,7]. DTPw-HBV/ $\mathrm{Hib}_{2.5}$, which contains a reduced amount of the purified polyribosyl-ribitol-phosphate capsular polysaccharide (PRP) of Hib covalently bound to tetanus toxoid has been subsequently developed. Furthermore, a formulation of this vaccine [(DTPw-HBV/Hib 2.5 [Kft]; Zilbrix(tm)-Hib)] manufactured at a new production site in Hungary and represented by ' $\mathrm{Kft}$ ' has been introduced.

The primary aim of this study was to demonstrate that (DTPw-HBV/Hib $2.5[\mathrm{Kft}]$ ) was not inferior to the licensed DTPw-HBV/Hib (Tritanrix(tm)-HepB/Hiberix(tm)) vaccine or the $\mathrm{DTPw}-\mathrm{HBV} / \mathrm{Hib}_{2.5}$ vaccine with respect to the immune response to the PRP antigen, when administered to healthy infants according to the Expanded Programme for Immunization (EPI) schedule at 6, 10 and 14 weeks of age. The DTPw-HBV/Hib 2.5 [Kft] differs from the other two vaccines in the study as it has components manufactured at a site in Hungary.

\section{Methods}

\section{Study design and subjects}

This phase III, observer-blind, randomized, primary vaccination study took place at three centres in India between June 2007 and January 2008. The trial followed the principles of the Declaration of Helsinki, and was compliant with the international standards of Good Clinical Practice and the local Indian Council of Medical Research guidelines governing clinical trials [8]. The study protocol was approved by the office of the Drugs Controller General of India (DCGI). The protocol was also subject to an institutional ethics committee review at each centre. Additionally, the study processes were subject to a sponsor audit without any critical findings.

Written, informed consent was obtained from parents/ guardians before enrolment. Healthy infants aged 6-8 weeks who had received one dose of the Hep $B$ vaccine within one week of birth were randomised to receive the DTPw-HBV/Hib ${ }_{2.5}[\mathrm{Kft}], \mathrm{DTPw}-\mathrm{HBV} / \mathrm{Hib}_{2.5}$ or DTPw-HBV/Hib vaccines at 6,10 and 14 weeks of age by intramuscular injection in the thigh.

\section{Vaccines}

The diphtheria and tetanus antigens of the DTPw-HBV/ $\mathrm{Hib}_{2.5}[\mathrm{Kft}]$ vaccine were produced at GSK Biologicals,
Korlatolt Felelossegu Tarsasag in Hungary, all other components of this, and the other vaccines, were developed and manufactured by GSK Biologicals, Rixensart, Belgium. The pertussis components were produced by the Commonwealth Serum Laboratory in Australia.

All vaccines contained: at least 30 international units (IU) of diphtheria toxoid, $60 \mathrm{IU}$ of tetanus toxoid and 4 IU of Bordetella pertussis (BPT), killed; and $10 \mu \mathrm{g}$ of hepatitis B surface antigen (HBsAg). The study vaccine, $\mathrm{DTPw}-\mathrm{HBV} / \mathrm{Hib}_{2.5}[\mathrm{Kft}]$ and the comparator DTPw$\mathrm{HBV} / \mathrm{Hib}_{2.5}$ both contained $2.5 \mu \mathrm{g}$ of the $H$. influenzae type $\mathrm{b}$ capsular polysaccharide conjugated to $5-10 \mu \mathrm{g}$ of the tetanus toxoid, compared to $10 \mu \mathrm{g}$ of the H. influen$z a e$ type $\mathrm{b}$ capsular polysaccharide conjugated to 20 to $40 \mu \mathrm{g}$ tetanus toxoid in the $\mathrm{DTPw}-\mathrm{HBV} / \mathrm{Hib}$ vaccine.

\section{Assessment of immunogenicity}

Blood samples were collected before the first dose of study vaccine and one month after the third vaccine dose and were tested for antibodies against all vaccine antigens, using enzyme-linked immunosorbent assays (ELISAs).

Anti-PRP antibodies were measured by ELISA with a cut-off set at $0.15 \mu \mathrm{g} / \mathrm{ml}$. Anti-diphtheria and anti-tetanus antibody concentrations were measured by ELISA with an assay cut-off set at $0.10 \mathrm{IU} / \mathrm{ml}$. Subjects seronegative for anti-diphtheria antibodies by ELISA were re-tested with an in vitro neutralization assay on Vero cells (cut-off of $0.016 \mathrm{IU} / \mathrm{ml}$ ). Anti-HBs antibodies were determined using either a commercial radioimmunoassay (AUSAB, Abbott), an ELISA developed in house or combination of the two, with an assay cut-off set at $10 \mathrm{mIU} / \mathrm{ml}$. Seroprotection was defined as antibody concentrations greater or equal to the assay cut-off. Anti-whole-cell-Bordetella Pertussis (BPT) antibody concentrations were measured by ELISA (AniLabsystems) with an assay cut-off set at $15 \mathrm{ELU} / \mathrm{ml}$.

\section{Assessment of Reactogenicity}

Reactogenicity was assessed using diary cards during a 4-day follow-up period after each vaccination. Reports of local symptoms of pain, redness and swelling at the site of injection, and of general symptoms of drowsiness, fever (defined as an axillary temperature $\geq 37.5^{\circ} \mathrm{C}$ ), irritability and loss of appetite were actively solicited. Symptom intensities for pain, irritability, drowsiness and loss of appetite were graded by the investigators on a three point scale. Grade 3 was defined as: cries when limb is moved/spontaneously painful (pain); diameter $>20 \mathrm{~mm}$ (swelling and redness); interfering with normal activities (other symptoms).

Unsolicited symptoms were recorded during the 30-day follow-up period after each vaccine dose and serious adverse events (SAE) were recorded throughout the duration of the trial. 


\section{Statistical Analysis}

The primary objective of the study was to sequentially assess and check the non-inferiority of the DTPw-HBV/ $\mathrm{Hib}_{2.5}[\mathrm{Kft}$ ] vaccine versus first DTPw-HBV/Hib and then DTPw-HBV/Hib 2.5 , in terms of the anti-PRP antibody response, after a three-dose primary vaccination course administered to healthy infants at 6,10 and 14 weeks of age.

The analysis of immunogenicity was based on the according to protocol (ATP) cohort for analysis of immunogenicity, which included all evaluable subjects for whom at least one measurement for the immunogenicity endpoint measures were available and who followed the study procedures. Evaluable subjects were those that met all the eligibility criteria, complied with the procedures defined in the protocol and were not eliminated from the study.

It was calculated that a sample size of 90 evaluable subjects per group would provide an overall power of $92 \%$ to meet the non-inferiority criteria for both the primary endpoints assuming the groups elicited identical immune responses. Allowing for an attrition rate of $10 \%$, a target sample size of 300 subjects (100 subjects per vaccine group) was selected.

Antibody seroprotection rates were calculated with 95\% confidence intervals (CIs) one month after the third vaccine dose. Geometric mean concentrations (GMCs) were calculated by taking the anti-log of the mean of the log concentration transformations. Antibody concentrations below the cut-off of the assay were given an arbitrary value of half the cut-off for the purpose of GMC calculation. As there is no established correlate of protection against pertussis, a vaccine response was defined as the appearance of antibodies in initially seronegative subjects, or maintenance or increase of prevaccination antibody concentrations in subjects that were seropositive prior to vaccination, taking into account the decline of maternal antibodies. All CIs calculated were 2-sided and computed using Proc StatXact SAS 9.1 and Proc StatXact 5 procedure on SAS. Noninferiority was considered to be reached if the upper limit of the two-sided $95 \% \mathrm{CI}$, for the differences in the percentage of subjects with anti-PRP antibody concentrations of $\geq 0.15 \mu \mathrm{g} / \mathrm{ml}$, was below the pre-defined limit of $10 \%$ one month after the $3^{\text {rd }}$ vaccine dose. Additional exploratory analyses of seroprotection using $95 \%$ CI on differences in seroprotection or vaccine response rates or ratios of GMCs between the study vaccine and comparator groups were undertaken.

The safety analysis was based on the Total Vaccinated Cohort. The incidence of solicited local and general adverse events (any or grade 3 intensity) was calculated using exact 95\% CI. Exploratory analyses were used to compare the incidence of solicited symptoms between the groups. The standardized asymptotic $95 \%$ CI for the difference between groups was also computed (StatXact 7) for the occurrence of solicited symptoms (during the 4-day follow-up period) after each vaccine dose, and overall per dose and per subject. CIs excluding zero indicated that a difference between groups may exist.

\section{Results}

A total of 300 subjects were recruited, of whom 299 subjects were vaccinated and included in the total vaccinated cohort and 267 were included in the ATP immunogenicity cohort of subjects (Figure 1). There were no withdrawals due to adverse events and 273 subjects completed the study. The demographic characteristics of the subjects in the three groups were similar (Table 1).

\section{Immunogenicity}

One month after the third vaccine dose, all subjects vaccinated with $\mathrm{DTPw}-\mathrm{HBV} / \mathrm{Hib}_{2.5}[\mathrm{Kft}]$ and DTPw$\mathrm{HBV} / \mathrm{Hib}$ and $98.8 \%$ of those vaccinated with DTPw$\mathrm{HBV} / \mathrm{Hib}_{2.5}$ had seroprotective levels of anti-PRP antibodies across all three groups (Table 2). The upper limit of the $95 \% \mathrm{CI}$ for the difference between groups in the percentage of subjects with seroprotective anti-PRP antibody concentrations $(\geq 0.15 \mu \mathrm{g} / \mathrm{ml})$ was below the predefined limit of $10 \%$. Thus the study vaccine, DTPw$\mathrm{HBV} / \mathrm{Hib}_{2.5}$ [Kft], was shown to be non-inferior to the two comparator vaccines.

In all three groups, seroprotective antibody concentrations were attained in $100 \%$ of subjects for diphtheria, tetanus and hepatitis $B$ one month after the vaccination course. The vaccine response rate to the pertussis component, at the same time point, was at least $97.8 \%$ in each group (Table 3).

Additional exploratory analyses also indicated the non-inferiority of DTPw-HBV/Hib ${ }_{2.5}[\mathrm{Kft}]$ to DTPw$\mathrm{HBV} / \mathrm{Hib}$ and DTPw-HBV/Hib 2.5 in terms of seroprotection rates to diphtheria, tetanus and $\mathrm{HepB}$ antigens and of vaccine response to the pertussis component. Non-inferiority of DTPw-HBV/Hib 2.5 [Kft] to DTPw$\mathrm{HBV} / \mathrm{Hib}_{2.5}$ was also demonstrated for antibody GMCs to diphtheria, tetanus, HepB and PRP antigens and for DTPw-HBV/Hib ${ }_{2.5}[\mathrm{Kft}]$ compared to DTPw-HBV/Hib with respect to antibody GMCs to the diphtheria, tetanus and hepatitis $B$ antigens.

\section{Safety}

Pain was the most frequently observed local solicited symptom during the 4-day follow-up period (Figure 2). Analysis per subject showed that fever was the most frequently observed solicited general symptom for DTPw$\mathrm{HBV} / \mathrm{Hib}_{2.5}$ [Kft] (80.2\%) and DTPw-HBV/Hib 2.5 (76.0\%) subjects, while irritability was the most frequently observed solicited general symptom for DTPw- 


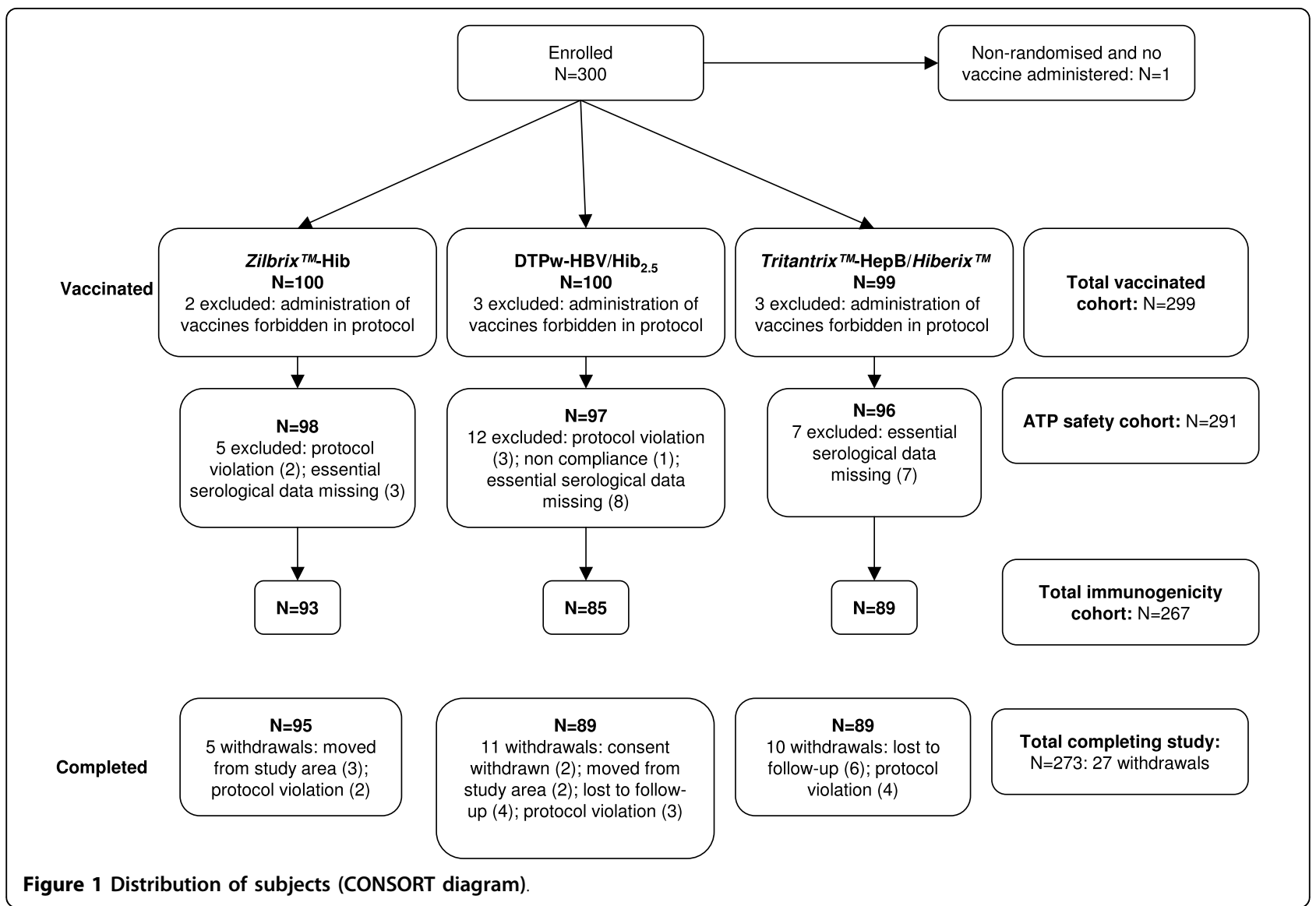

Table 1 Summary of demographic characteristics (Total Vaccinated Cohort)

\begin{tabular}{|c|c|c|c|c|c|c|c|c|c|}
\hline \multirow{2}{*}{\multicolumn{2}{|c|}{ Characteristics }} & \multicolumn{2}{|c|}{ DTPw-HBV/Hib $2.5[K f t](N=100)$} & \multicolumn{2}{|c|}{ DTPw-HBV/Hib (N = 99) } & \multicolumn{2}{|c|}{ DTPw-HBV/Hib2.5 $(\mathrm{N}=100)$} & \multicolumn{2}{|c|}{ Total $(\mathrm{N}=299)$} \\
\hline & & Value or $n$ & $\%$ & Value or $n$ & $\%$ & Value or $\mathbf{n}$ & $\%$ & Value or $n$ & $\%$ \\
\hline \multirow[t]{2}{*}{ Age (weeks) } & Mean & 6.3 & - & 6.3 & - & 6.4 & - & 6.3 & - \\
\hline & SD & 0.59 & - & 0.51 & - & 0.64 & - & 0.58 & - \\
\hline \multirow[t]{2}{*}{ Weight (kg) } & Mean & 3.9 & - & 3.9 & - & 3.8 & - & 3.9 & - \\
\hline & SD & 0.69 & - & 0.66 & - & 0.70 & - & 0.69 & - \\
\hline \multirow[t]{2}{*}{ Gender } & Female & 39 & 39.0 & 37 & 37.4 & 48 & 48.0 & 124 & 41.5 \\
\hline & Male & 61 & 61.0 & 62 & 62.6 & 52 & 52.0 & 175 & 58.5 \\
\hline Race & Asian & 100 & 100.0 & 99 & 100.0 & 100 & 100.0 & 299 & 100.0 \\
\hline
\end{tabular}

$\mathrm{N}=$ total number of subjects; $\mathrm{n} / \%=$ number/percentage of subjects in a given category; $\mathrm{SD}=$ standard deviation.

Table 2 Anti-PRP antibody response before dose 1 (pre) and one month post-dose 3 (post; ATP cohort for immunogenicity)

\begin{tabular}{|c|c|c|c|c|c|c|c|c|}
\hline & \multirow[t]{2}{*}{ Time point } & \multirow[b]{2}{*}{$\mathbf{N}$} & \multicolumn{2}{|c|}{$\geq 0.15 \mu \mathrm{g} / \mathrm{mL}$} & \multicolumn{2}{|c|}{$\geq 1.0 \mu \mathrm{g} / \mathrm{mL}$} & \multicolumn{2}{|c|}{ GMC } \\
\hline & & & $\%$ & $95 \% \mathrm{Cl}$ & $\%$ & $95 \% \mathrm{Cl}$ & Value & $95 \% \mathrm{Cl}$ \\
\hline \multirow[t]{2}{*}{ DTPw-HBV/Hib ${ }_{2.5}[\mathrm{Kft}]$} & Pre & 93 & 44.1 & $33.8-54.8$ & 9.7 & $4.5-17.6$ & 0.179 & $0.139-0.230$ \\
\hline & Post & 93 & 100 & $96.1-100$ & 94.6 & $87.9-98.2$ & 26.71 & $19.161-37.23$ \\
\hline \multirow[t]{2}{*}{ DTPw-HBV/Hib } & Pre & 89 & 42.7 & $32.3-53.6$ & 10.1 & $4.7-18.3$ & 0.177 & $0.137-0.227$ \\
\hline & Post & 89 & 100 & $95.9-100$ & 100 & $95.9-100$ & 40.75 & $32.301-51.40$ \\
\hline \multirow[t]{2}{*}{ DTPw-HBV/Hib 2.5} & Pre & 84 & 42.9 & $32.1-54.1$ & 8.3 & $3.4-16.4$ & 0.167 & $0.131-0.212$ \\
\hline & Post & 85 & 98.8 & 93.6-100 & 94.1 & 86.8-98.1 & 19.58 & $14.019-27.36$ \\
\hline
\end{tabular}

$\mathrm{N}=$ total number of subjects; $\mathrm{Cl}=$ confidence interval; $\mathrm{GMC}=$ geometric mean concentration. 
Table 3 Seroprotection rates and GMCs for diphtheria, tetanus, HBV and pertussis before dose 1 (pre) and one month post-dose 3 (post; ATP cohort for immunogenicity)

\begin{tabular}{|c|c|c|c|c|c|c|c|c|c|c|c|c|c|c|c|c|}
\hline & \multirow[b]{3}{*}{ Time point } & \multicolumn{9}{|c|}{ Seroprotection rates/Vaccine response rates } & \multicolumn{6}{|c|}{ GMC } \\
\hline & & \multicolumn{3}{|c|}{$D T P w-H B V / H i b_{2.5}[K f t]$} & \multicolumn{3}{|c|}{$D T P w-H B V / H i b$} & \multicolumn{3}{|c|}{$D T P w-H B V / H i b_{2.5}$} & \multicolumn{2}{|c|}{$\mathrm{DTPw}-\mathrm{HBV} / \mathrm{Hib}{ }_{2.5}[\mathrm{Kft}]$} & \multicolumn{2}{|c|}{$D T P w-H B V / H i b$} & \multicolumn{2}{|c|}{$D T P w-H B V / H i b_{2.5}$} \\
\hline & & $\mathbf{N}$ & $\%$ & $95 \% \mathrm{Cl}$ & $\mathrm{N}$ & $\%$ & $95 \% \mathrm{Cl}$ & $\mathbf{N}$ & $\%$ & $95 \% \mathrm{Cl}$ & Value & $95 \% \mathrm{Cl}$ & Value & $95 \% \mathrm{Cl}$ & Value & $95 \% \mathrm{Cl}$ \\
\hline \multirow{2}{*}{$\begin{array}{l}\text { Diphtheria } \geq \mathbf{0 . 1} \mathbf{I U} / \mathrm{ml} \text { (or } \\
\geq 0.016 \mathrm{IU} / \mathrm{ml} \text { by } \\
\text { neutralization assay) }\end{array}$} & Pre & 93 & 31.2 & $22.0-41.6$ & 89 & 34.8 & $25.0-45.7$ & 85 & 24.7 & $16.0-35.3$ & 0.086 & $0.070-0.105$ & 0.082 & $0.069-0.097$ & 0.076 & $0.063-0.093$ \\
\hline & Post & 93 & 100 & $96.1-100$ & 89 & 98.9 & $95.9-100$ & 85 & 100 & $95.8-100$ & 2.89 & $2.30-3.64$ & 1.76 & $1.41-2.19$ & 1.65 & $1.36-2.02$ \\
\hline \multirow[t]{2}{*}{ Tetanus $\geq 0.1 \mathrm{IU} / \mathrm{ml}$} & Pre & 93 & 100 & $96.1-100$ & 89 & 100 & $95.9-100$ & 85 & 100 & $95.8-100$ & 1.950 & $1.561-2.437$ & 2.416 & $1.991-2.932$ & 2.112 & $1.751-2.548$ \\
\hline & Post & 93 & 100 & $96.1-100$ & 89 & 100 & $95.9-100$ & 85 & 100 & $95.8-100$ & 4.74 & $3.78-5.95$ & 2.82 & $2.32-3.45$ & 2.77 & $2.24-3.44$ \\
\hline \multirow[t]{2}{*}{$\mathrm{HBV} \geq 10 \mathrm{mIU} / \mathrm{ml}$} & Pre & 85 & 25.9 & $17.0-36.5$ & 85 & 24.7 & $16.0-35.3$ & 84 & 21.4 & $13.2-31.7$ & 8.3 & $6.7-10.3$ & 8.0 & $6.5-9.8$ & 6.6 & $5.8-7.5$ \\
\hline & Post & 92 & 100 & $96.1-100$ & 89 & 100 & $95.9-100$ & 85 & 100 & $95.8-100$ & 781.1 & $629.7-968.8$ & 598.2 & 477.7-749.1 & 695.3 & $542.1-891.7$ \\
\hline \multirow{2}{*}{$\begin{array}{l}\text { Anti-BPT antibodies } \geq 15 \text { El. } \\
\mathrm{U} / \mathrm{ml}\end{array}$} & Pre & 93 & 16.1 & $9.3-25.2$ & 89 & 19.1 & $11.5-28.8$ & 85 & 22.4 & $14.0-32.7$ & 9.1 & 8.2-10.1 & 9.1 & 8.3-10.0 & 9.8 & 8.7-11.0 \\
\hline & Post & 93 & 98.9 & $94.2-100$ & 89 & 98.9 & $93.9-100$ & 85 & 100 & $95.8-100$ & 63.4 & $55.4-72.7$ & 83.7 & $73.6-95.2$ & 100.3 & 88.4-113.7 \\
\hline VR for anti-BPT antibody & Post & 93 & 97.8 & $92.4-99.7$ & 89 & 98.9 & 93.9-100 & 85 & 98.8 & $93.6-100$ & & & & & & \\
\hline
\end{tabular}

$\mathrm{BPT}=$ Bordetella pertussis; $\mathrm{Cl}=$ confidence interval; $\mathrm{GMC}=$ geometric mean concentration; $\mathrm{HBV}=$ hepatitis $\mathrm{B}$ vaccine; $\mathrm{IU}=$ international unit; $\mathrm{N}=$ number of subjects with available results; $\mathrm{VR}=$ vaccine response. 


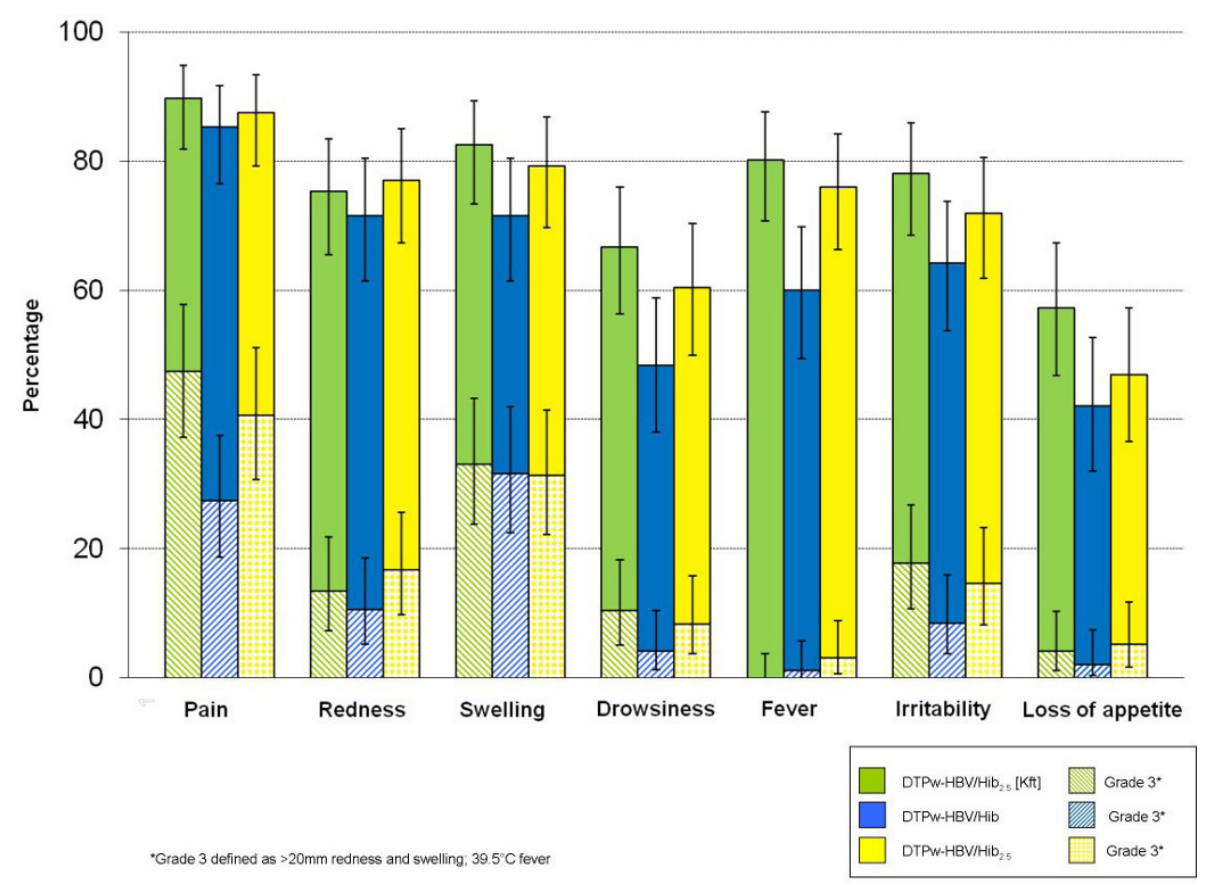

Figure 2 Incidence of solicited local and general adverse events, within 4 days of vaccination after all doses (Total Vaccinated Cohort).

HBV/Hib (64.2\%). A higher overall incidence of grade 3 adverse events was observed in the DTPw-HBV/Hib 2.5 [Kft] group.

The percentage of subjects reporting at least one unsolicited symptom within the 31 day follow up period after the administration of any vaccination dose was less than $6 \%$ in any of the groups. None of the subjects reported grade 3 unsolicited symptoms related to vaccination.

No SAEs were reported in any of the groups.

\section{Discussion}

Vaccination of Indian infants against diphtheria, tetanus and pertussis is recommended according to the EPI schedule at 6, 10 and 14 weeks [9]. In addition, the Indian Academy of Pediatrics and WHO [10] recommend a birth dose of HBV to reduce the vertical transmission of hepatitis B to newborns, as well as HBV priming doses at the EPI time points. In 2006, WHO recommended the worldwide incorporation of Haemophilus influenzae type b vaccination into all routine infant immunization programs from as early as possible after 6 weeks of age [11]. In India Hib priming doses at the EPI time points are recommended.

Co-administration of multiple antigens in a single injection is widely accepted as beneficial with respect to convenience, compliance and timeliness of vaccination $[1,2,12]$. With respect to the DTPw-HBV-Hib vaccines, many clinical studies have shown that the five antigens can be co-administered without impacting upon the immunogenicity of the individual components [13]. The combination vaccine, DTPw-HBV-Hib, containing $10 \mu \mathrm{g}$ PRP was licensed for primary and booster immunization of Indian infants in 2000 and has been locally shown to be immunogenic and well tolerated [14].

This trial studied the administration of the DTPw$\mathrm{HBV} / \mathrm{Hib}_{2.5}[\mathrm{Kft}]$ vaccine according to the challenging 6-10-14 week EPI schedule, with prior administration of a birth dose of $\mathrm{HBV}$, as recommended locally by the IAP and the WHO.

All subjects were seroprotected against Hib, diphtheria, tetanus and HBV one month after the third dose and $97.8 \%$ subjects showed a vaccine response to pertussis. These findings are similar to those achieved by the licensed vaccine, and comparable to levels previously reported in studies using these pentavalent vaccines in an Asian population [5,6,14-16]. Our study demonstrated DTPw-HBV/Hib $2.5[\mathrm{Kft}]$ to be noninferior in terms anti-PRP antibody response compared to both DTPw-HBV/Hib vaccine and DTPw-HBV/Hib 2.5 vaccine after a three-dose primary vaccination course. However, the anti-PRP GMC point estimate for the comparator DTPw-HB/Hib 2.5 vaccine (which, unlike the other 2 vaccines [Tritanrix(tm)-HepB/Hiberix(tm) and Zilbrix-Hib(tm)] used in this study, is not a WHO prequalified) vaccine $[17,18]$ was significantly lower than for the full-content DTPw-HB/Hib. The results from this study agree with previous findings from the 
Philippines and Latina comparing a DTPw- $\mathrm{HB} / \mathrm{Hib}_{2.5}$ vaccine with the licensed vaccine $[6,19]$ and support the observation that vaccines containing reduced amounts of PRP-tetanus toxoid conjugate generate effective antibody responses and immunological protection $[16,7,20-26]$. The responses to the other antigens of DTPw-HBV/Hib 2.5 [Kft] were also found to be within similar $95 \%$ confidence intervals as the other vaccines, in terms of seroprotection and vaccine response, further substantiating the non-inferiority of the DTPw-HBV/ $\mathrm{Hib}_{2.5}[\mathrm{Kft}]$ vaccine to the two comparator vaccines.

Overall, the safety profile of the new DTPw-HBV/ $\mathrm{Hib}_{2.5}$ [Kft] vaccine appeared to be similar to that of the licensed comparator vaccines and the incidence of symptoms reported was within the range of incidences reported in the literature after DTPw-based combination vaccines [5].

This study was designed to assess primary vaccination at 6,10 and 14 weeks. In clinical practice an additional booster dose of the vaccine is recommended at 12-18 months. Although a booster was not included in this study, the vaccine was immunogenic (seroprotection above accepted minimum cut-off values) following the primary vaccination course. The non-inferiority analysis was not performed on the PRP cut-off $\geq 1.0 \mu \mathrm{g} / \mathrm{mL}$, as the study was not powered for this comparison, although this concentration is regarded as an indicator of long-term protection. Nevertheless, the CIs for the percentage of subjects seroprotected overlapped (at least $94.1 \%$ in all groups) indicating that all three vaccines conferred long-term protection. In addition, The Hib immune response to the DTPw$\mathrm{HBV} / \mathrm{Hib}_{2.5}[\mathrm{Kft}]$ was similar to that observed with another, WHO prequalified, DTPw-HBV-Hib vaccine administered in a 2-3-4 month schedule [27].

Reduction of the antigen content of the Hib component of DTPw-HBV/Hib and the different formulation of the DTPw-HBV/Hib $2.5[\mathrm{Kft}]$ DTPw antigens did not have any significant effects on the safety, reactogenicity and immunogenicity parameters tested of the combination vaccine when compared to the licensed comparator vaccines. However, further studies need to be carried out to assess any interference on other vaccines, which may be co-administered at the EPI time points, such as rotavirus vaccine or conjugated pneumococcus vaccine.

\section{Conclusions}

The reduced PRP, new formulation, DTPw-HBV/Hib 2.5 [Kft] vaccine was immunogenic and well tolerated when administered according to the EPI schedule to Indian infants. It was also shown to be non-inferior to DTPw$\mathrm{HBV} / \mathrm{Hib}$ and DTPw-HBV/Hib 2.5 in terms of seroprotection rates to diphtheria, tetanus and $\mathrm{HepB}$ antigens and of vaccine response to the pertussis component.

\section{Abbreviations}

ATP: (according to protocol); BPT: (Bordetella Pertussis); Cl: (confidence interval); DTPw: (diphtheria-tetanus-pertussis); EPI: (Expanded Programme for Immunization); GMC: (geometric mean concentration); GSK:

(GlaxoSmithKline); HBsAg: (hepatitis B surface antigen); HBV: (hepatitis B vaccine); Hep B: (hepatitis B); Hib: (Haemophilus influenza type b); IAP: (Indian Academy of Pediatrics); IU: (international unit); rHBsAg: (recombinant HBsAg); PRP: (polyribosyl-ribitol-phosphate); Pw: (whole cell pertussis); SAE: (serious adverse events); WHO: (World Health Organization).

\section{Acknowledgements}

The authors would like to acknowledge the contributions of Dr. Dipak Patra, Dr. Anand Prakash and Dr. Vineeta Gupta, who were investigators at the Medical College Kolkata, St John's Medical College Hospital, Bangalore, and Institute of Medical Sciences, Banaras Hindu University, Varanasi, respectively. We also thank Emma Beatty and Julia Donnelly for providing editorial support during the development of this manuscript.

Zilbrix, Hiberix and Tritanrix are trademarks of the GlaxoSmithKline group of companies.

\section{Author details}

${ }^{1}$ Department of Pediatrics, Medical College Kolkata, Kolkata, India. ${ }^{2}$ St John's Medical College Hospital, Bangalore, India. ${ }^{3}$ Department of Pediatrics, Institute of Medical Sciences, Banaras Hindu University, Varanasi, India. ${ }^{4}$ GlaxoSmithKline Biologicals, Bangalore, India and Wavre, Belgium.

\section{Authors' contributions}

The corresponding author had full access to the data and was responsible for submission of the publication.

All authors participated in the design or implementation, analysis and interpretation of the study, the writing of the report and the decision to submit the manuscript for publication.

\section{Competing interests}

GlaxoSmithKline Biologicals was the funding source and was involved in all stages of the study conduct and analysis. GSK Biologicals also funded all costs associated with the development and the publishing of the present manuscript.

Sanjoy Datta, Jeanne-Marie Jacquet and Alix Collard are all employed by GlaxoSmithKline.

Received: 19 March 2010 Accepted: 15 October 2010

Published: 15 October 2010

\section{References}

1. Decker MD: Principles of pediatric combination vaccines and practical issues related to clinical practice. Pediatr Infect Dis J 2001, 20(11 Suppl): S10-18.

2. Kalies H, Grote V, Verstraeten T, Hessel L, Schmitt HJ, von Kries R: The use of combination vaccines has improved timeliness of vaccination in children. Pediatr Infect Dis J 2006, 25:507-12.

3. World Health Organization and UNICEF: Global immunization coverage. 2009 [http://www.who.int/immunization/newsroom/GID_english.pdf], Last accessed April 1st 2009.

4. Chunsuttiwat S, Biggs BA, Maynard JE, Thammapormpilas P, OPrasertsawat M: Comparative evaluation of a combined DTP-HB vaccine in the EPI in Chiangrai Province, Thailand. Vaccine 2002, 21:188-93.

5. Arístegui J, Usonis V, Coovadia H, Riedemann S, Win KM, Gatchalian S, Bock HL: Facilitating the WHO expanded program of immunization: the clinical profile of a combined diphtheria, tetanus, pertussis, hepatitis $B$ and Haemophilus influenzae type b vaccine. Int J Infect Dis 2003, 7:143-51.

6. Gatchalian S, Reyes M, Bernal N, Lefevre I, David MP, Han HH, Bock HL, Wolter J, Schuerman L: A new DTPw-HBV/Hib vaccine is immunogenic and safe when administered according to the EPI (Expanded Programme for Immunization) schedule and following hepatitis B vaccination at birth. Human Vaccines 2005, 1:198-203.

7. Lagos R, Valenzuela MT, Levine OS, Losonsky GA, Erazo A, Wasserman SS, Levine MM: Economisation of vaccination against Haemophilus influenzae type b: a randomised trial of immunogenicity of fractionaldose and two-dose regimens. Lancet 1998, 351(9114):1472-6. 
8. ICMR: Indian Council of Medical Research guidelines governing clinical trials. 2006 [http://icmr.nic.in/ethical_guidelines.pdf].

9. World Health Organization: Immunization Policy. Global Programme for Vaccines and Immunization, Expanded Programme on Immunization. 1996, WHO/EPI/GEN/95.03REV.1.

10. World Health Organization: Introduction of hepatitis B vaccine into childhood immunization services. 2001 [http://www.who.int/ immunization_delivery/publications/HepB-MG_English.pdf], Last accessed 1st April 2009.

11. World Health Organization: WHO position paper on Haemophilus influenzae type b conjugate vaccines. Wkly Epidemiol Rec 2006, 81(47):445-52.

12. Marshall GS, Happe LE, Lunacsek OE, Szymanski MD, Woods CR, Zahn M, Russell A: Use of combination vaccines is associated with improved coverage rates. Pediatr Infect Dis J 2007, 26(6):496-500.

13. Prymula R, Plisek S: Clinical experience with DTPw-HBV and DTPw-HBV/ Hib combination vaccines. Exp Opin Biol Ther 2008, 8(4):503-13.

14. Bavdekar SB, Maiya PP, Subba Rao SD, Datta SK, Bock HL: Immunogenicity and safety of combined diphtheria-tetanus-whole cell pertussis-hepatitis $B /$ Haemophilus influenzae type $b$ vaccine in Indian infants previously primed at birth with hepatitis B vaccination. Indian Pediatrics 2007, 44(7):505-10.

15. Bravo L, Carlos J, Gatchalian S, Borja-Tabora C, Bibera G, Willems P, Safary A, Bock HL: The new DTPw-HBV-Hib combination vaccine can be used at the WHO schedule with a monovalent dose of hepatitis B vaccine at birth. Southeast Asian J Trop Med Public Health 1998, 29:772-8.

16. Hla KH, Thein SA, Aye A, Han HH, Bock HL, David MP, Schuerman L: Reactogenicity and immunogenicity profiles of a novel pentavalent diphtheria-tetanus-whole cell pertussis-hepatitis B and Haemophilus influenzae type $b$ vaccine: a randomized dose-ranging trial of the Hib tetanus-conjugate content. Pediatr Infect Dis J 2006, 25:706-12.

17. World Health Organisation: Immunization Standards. Diphtheria-TetanusPertussis (whole cell)-Hepatitis B-Haemophilus influenzae type b (1 dose vial). [http://www.who.int/immunization_standards/vaccine_quality/ 56_diphteta/en/index.html], Last accessed 12th July 2010..

18. World Health Organisation: Immunization Standards. Diphtheria-TetanusPertussis (whole cell)-Hepatitis B-Haemophilus influenzae type $b$ (1 dose vial). [http://www.who.int/immunization_standards/vaccine_quality/ 59_diphteta/en/index.html], Last accessed 12th July 2010.

19. Espinoza F, Tregnaghi M, Gentile A, Abarca K, Casellas J, Collard A, Lefevre I, Jacquet J-M: Primary and booster vaccination in Latin American children with a DTPW-HBV/Hib combination: a randomized controlled trial. BMC Infect Dis 2010.

20. Fernandez J, Balter S, Feris J, Gomez E, Garib Z, Castellanos PL, Sánchez J, Romero-Steiner $S$, Levine OS: Randomized trial of the immunogenicity of fractional dose regimens of PRP-T Haemophilus influenzae type $b$ conjugate vaccine. Am J Trop Med Hyg 2000, 62:485-90.

21. Romero-Steiner S, Fernandez J, Biltoft C, Wohl ME, Sanchez J, Feris J, Balter S, Levine OS, Carlone GM: Functional antibody activity elicited by fractional doses of Haemophilus influenzae type $b$ conjugate vaccine (polyribosylribitol phosphate-tetanus toxoid conjugate). Clin Diagn Lab Immunol 2001, 8:1115-9.

22. Campbell JD, Lagos R, Levine MM, Losonosky GA: Standard and alternative regimens of Haemophilus influenzae type $b$ conjugate vaccine (polyribosylribitol phosphate-tetanus toxoid conjugate vaccine) elicit comparable antibody avidities in infants. Pediatr Infect Dis J 2002, 21:822-6.

23. Huebner RE, Nicol M, Mothupi R, Kayhty H, Mbelle N, Khomo E, Klugman KP: Dose response of CRM197 and tetanus toxoid-conjugated Haemophilus influenzae type b vaccines. Vaccine 2004, 23:802-6.

24. Nicol M, Huebner R, Mothupi R, Käyhty H, Mbelle N, Khomo E: Haemophilus influenzae type $b$ conjugate vaccine diluted tenfold in diphtheria-tetanus-whole cell pertussis vaccine: a randomized trial. Paediatr Infect Dis J 2002, 21:138-41.

25. Tregnaghi M, Lopez P, Rocha C, Rivera L, David MP, Ruttimann R, Schuerman L: A new DTPW-HB/Hib combination vaccine for primary and booster vaccination of infants in Latin America. Rev Panam Salud Publica 2006, 19:179-88.

26. Gatchalian S, Reyes M, Bermal N, Chandrasekaran V, Han HH, Bock HL, Lefevre I: A new DTPw-HBV/Hib vaccine: immune memory after primary vaccination and booster dosing in the second year of life. Human Vaccines 2008, 4(1):60-6.

27. Kanra G, Kara A, Demiralp O, Contorni M, Hilbert AK, Spyr C, Viviani S: Safety and immunogenicity of a new fully liquid DTPW-HepB-Hib combination vaccine in infants. Hum Vacc 2006, 2(4):155-60.

\section{Pre-publication history}

The pre-publication history for this paper can be accessed here: http://www.biomedcentral.com/1471-2334/10/298/prepub

doi:10.1186/1471-2334-10-298

Cite this article as: Chatterjee et al: The immunogenicity and safety of a reduced PRP-content DTPW-HBV/Hib vaccine when administered according to the accelerated EPI schedule. BMC Infectious Diseases 2010 10:298.

\section{Submit your next manuscript to BioMed Central and take full advantage of:}

- Convenient online submission

- Thorough peer review

- No space constraints or color figure charges

- Immediate publication on acceptance

- Inclusion in PubMed, CAS, Scopus and Google Scholar

- Research which is freely available for redistribution

Submit your manuscript at www.biomedcentral com/submit
Ciomed Central 